\title{
Surgical and anaesthetic evaluation of neuter in guinea pig- 2 cases
}

\begin{abstract}
Guinea pigs (Cavia porcellus) play important role for biological and immunological research and sometimes as a pet. As a pet, castration is required for control the breeding and reduces the aggressiveness. The present case study represents the efficacy of surgical outcome and anaesthetic protocol. Two male English variety (short haired) guinea pig weighing 650gms and $400 \mathrm{gms}$ aged about 6 months was presented to the SAQ Teaching Veterinary Hospital, Chittagong Veterinary and Animal Sciences University (CVASU), Chittagong, Bangladesh, for castration. Both guinea pigs were active and alert. On clinico-andrological examination, both descended testes were found to be intact. The vital parameters viz., temperature, respiration rate and heart rate were recorded as $100^{\circ} \mathrm{F}, 56 / \mathrm{min}$ and 236 beats $/$ minute respectively. Open closed method castration was performed under aseptic condition with xylazine and ketamine anaesthesia with the dose rate $8 \mathrm{mg} / \mathrm{kg}$ and $60 \mathrm{mg} / \mathrm{kg}$ respectively. The guinea pigs became unconscious within two minutes of intramuscular injection and full recovered after 50 minutes of injection. Post operative treatment was maintained with antibiotic, painkiller for 5 days. Mild suppuration was noticed postoperatively 7 days and dressing was performed accordingly. The wound healing was noticed after 10 days of operation. The case report suggests that guinea pig castration can be performed successfully with xylazine and ketamine anaesthesia with recommended doses.
\end{abstract}

Keywords: guinea pig, evaluation neuter
Volume 8 Issue 6 - 2017

Saroj Kumar Yadav,' Md.Afzal Hossain,' Tuli Dey,' Mohammad Bayazid Bostami,' Bibek Chandra Sutradhar, ${ }^{2}$ Bhajan Chandra Das ${ }^{2}$ 'MS Student, Department of Medicine and Surgery, Chittagong Veterinary and Animal Sciences University (CVASU), Bangladesh 2Professor, Department of Medicine and Surgery, Chittagong Veterinary and Animal Sciences University (CVASU), Bangladesh

Correspondence: Saroj Kumar Yadav, MS Student, Department of Medicine and Surgery, Chittagong Veterinary and Animal Sciences University (CVASU), Bangladesh,

Emailshirfraaz@gmail.com

Received: April 07, 2017 | Published: October 12, 2017

\section{Introduction}

Guinea Pigs (cavia porcellus) are belonging to the family Caviidae and the genus Cavia. The Boars are very difficult to maintain in groups without fighting and grave physically damage, and neuter was used as a way of minimizing this violent behavior. ${ }^{1}$ The method described in this paper is simpler, Guinea pigs (Cavia porcellus) was handled softly and with concern to injury to the pet and the surgeon appropriately following hygiene as recommended by Mcgreevy. ${ }^{2}$ Mainly boars get sexual maturity by 4-6 weeks of age and are capable of impregnating the sows. ${ }^{3}$ Castration has been performed by open closed technique using tip of scrotal incision under general anesthesia. ${ }^{4}$

\section{Case history and description}

On January 11-2016, Two male English variety (short haired) guinea pig weighing $650 \mathrm{gms}$ and $400 \mathrm{gms}$ aged about 6 months was presented to the SAQ Teaching Veterinary Hospital, Chittagong Veterinary and Animal Sciences University (CVASU), Chittagong, Bangladesh, for castration. Both guinea pigs were active and alert. On clinico-andrological examination, both descended testes were found to be intact. The vital parameters viz., temperature, respiration rate and heart rate were recorded as $100^{\circ} \mathrm{F}, 56 / \mathrm{min}$ and 236 beats/minute respectively (Figure $1 \& 2$ ).

\section{Restraining anaesthesia with and close monitoring}

General anesthesia was obtained by injecting xylazine hydrochloride (Inj. Xylazine ${ }^{\circledR}-8 \mathrm{mg} / \mathrm{kg}$ body weight) intramuscularly followed by ketamine hydrochloride (Inj. G-ketamine ${ }^{\circledR}-60 \mathrm{mg} / \mathrm{kg}$ body weight) respectively. And regularly monitoring heart rate and eye reflexes.

\section{Surgical procedure}

The guinea pig was anaesthetized by intramuscular injection of $0.26 \mathrm{~mL}$ (@8 $8 \mathrm{mg} / \mathrm{kg}$ body weight xylazine (xylaxin ${ }^{2}, 20 \mathrm{mg} / \mathrm{mL}$,
India Immunologicals Ltd., Hyderabad, India), $0.75 \mathrm{~mL}$ ketamine (G-ketamine ${ }^{\circledR}, 60 \mathrm{mg} / \mathrm{mL}$, Gonoshasthaya Pharmaceuticals Ltd., Mirzanagar Dhaka, Bangladesh). The pet became unconscious within two minutes. As soon as the pet was anesthetized, during the preparation of surgical site and surgical procedure the pet was provided with warmth using towel wrap as the guinea pigs are sensitive to hypothermia while under anaesthesia. The temperature, heart rate and respiration are monitored throughout the procedure. The scrotal area of about $4 \mathrm{~cm}$ x $5 \mathrm{~cm}$ was shaved and cleaned (Figure $3 \& 4$ ).

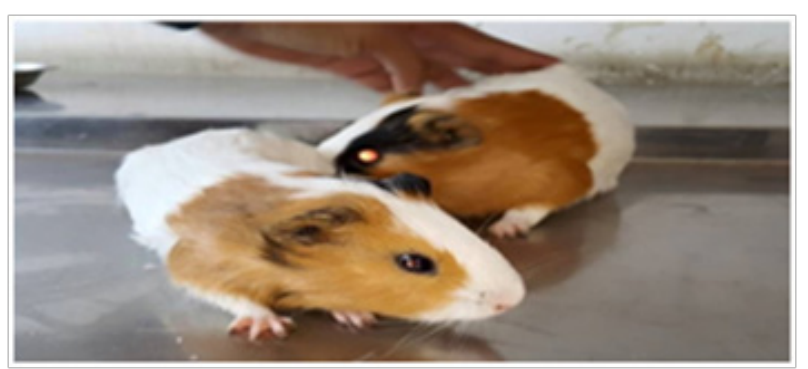

Figure I

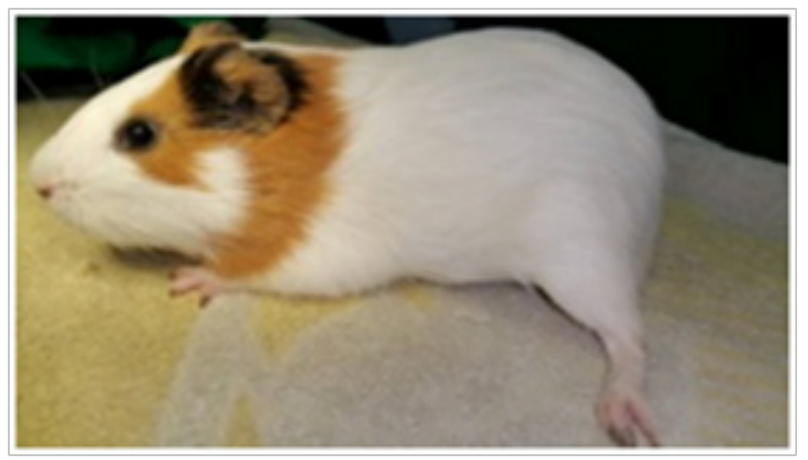

Figure 2 


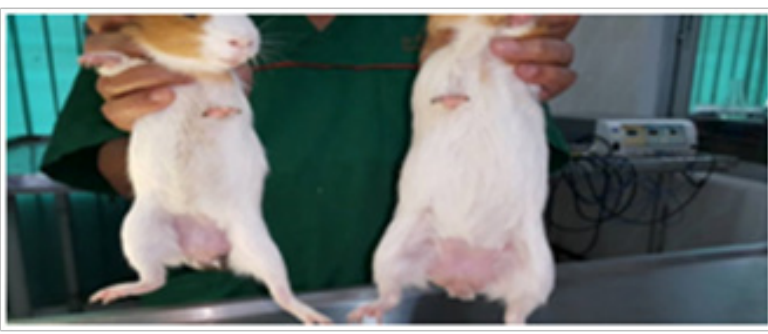

Figure 3

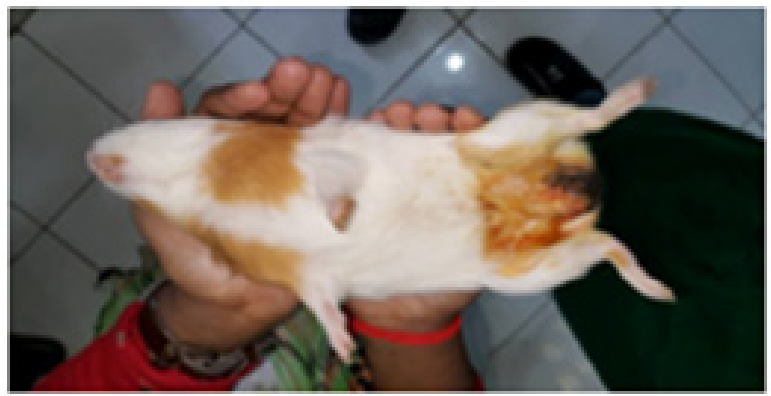

Figure 4

The surgical area was scrubbed with $7.5 \%$ povidone iodine solution. This was repeated for 3 times times. Later, when the pet was shifted to the operation theatre the area was again sterilized with $7.5 \%$ surgical povidone iodine solution and surgical spirit alternatively twice. Both the testes were manipulated from within the peritoneal cavity, through the inguinal ring and held tightly within the scrotum by fingers. An incision of one cm tip of the testis through the scrotal skin (Figure 5 \& 6).

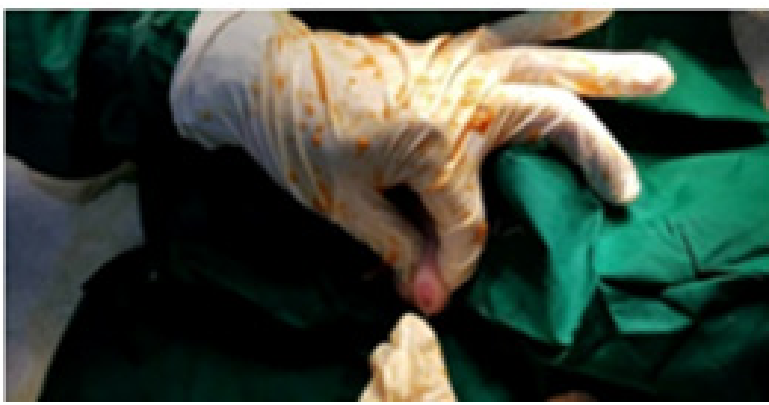

Figure 5

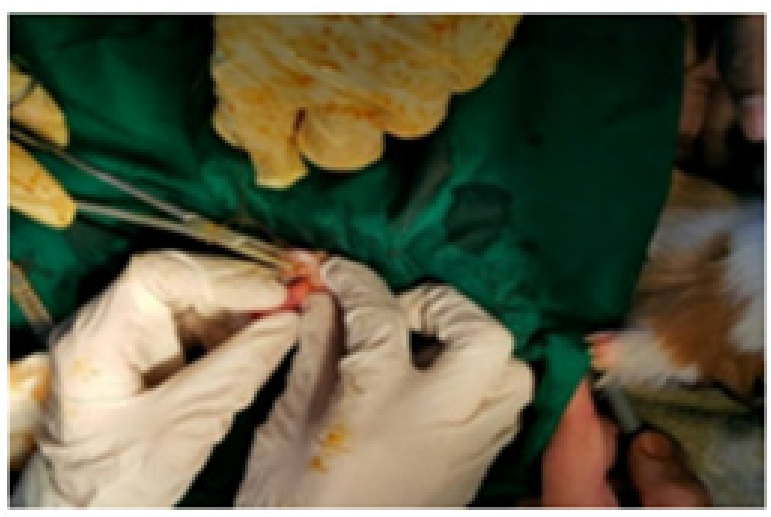

Figure 6
The tunica dartos was cut carefully leaving the tunica vaginalis intact. The digital pressure was applied to expose the testis to come through the incision. A small artery forceps/hemostat was applied on to the spermatic cord and a ligature of absorbable suture material, catgut No. 2.0 (Trugut ${ }^{\circledR}$, Suture India Pvt. Ltd., Bangalore, India) was applied proximal to the haemostat (Figure 7).

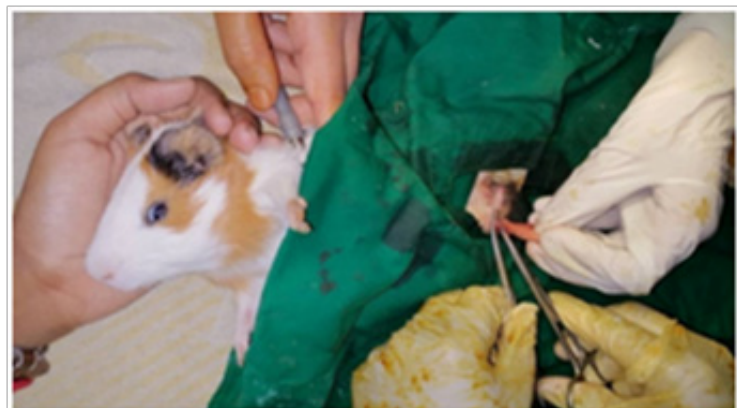

Figure 7

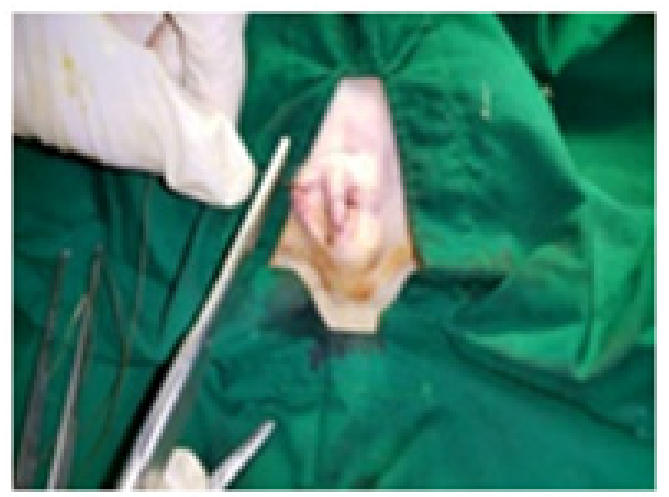

Figure 8

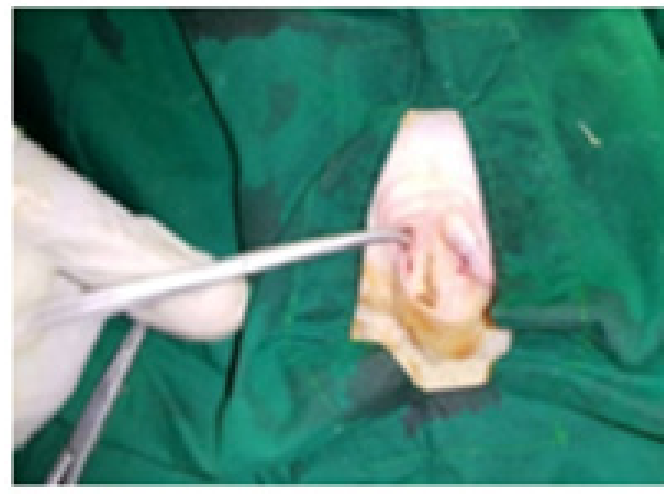

Figure 9

The knot was sufficiently tightened to ensure hemostasis and ligature retention and the hemostat was removed following excising the cord distal to it. Care was taken during ligating and cutting the cord to avoid any opening into the peritoneal cavity. The scrotal incision was closed using absorbable suture material, chromic catgut No. 2.0 with simple interrupted suture. The procedure was repeated for removal of the other testis (Figure $8 \&$ 9). Postoperatively, the surgical wound was dressed with $5 \%$ povidone iodine ointment (Povicep ${ }^{\circledR}$, Jason Ltd., Dhaka, Bangladesh). The pet was shifted to the recovery and ventilation room to aid in comfortable recovery (Figure 10). 


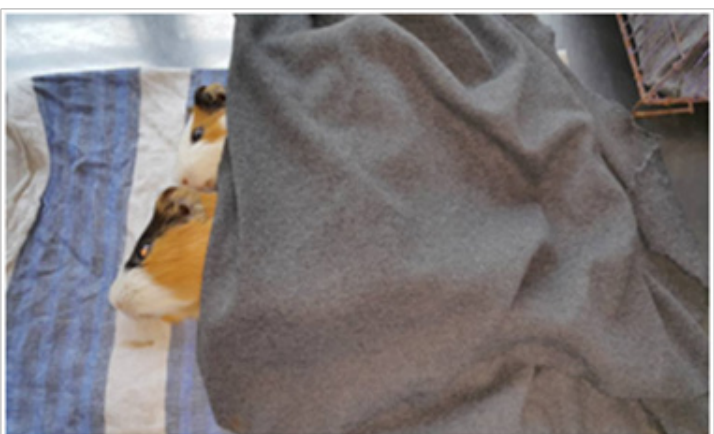

Figure 10

Recovery occurs as: Time of anesthesia was 11.30 AM

\begin{tabular}{ll}
\hline Mouth reflex & $1.30 \mathrm{pm}$ \\
Eye reflex & $3 \mathrm{pm}$ \\
Come in sitting position & $4.10 \mathrm{pm}$ \\
Start working & $4.45 \mathrm{pm}$ \\
Start normal feeding & $5 \mathrm{pm}$ \\
\hline
\end{tabular}

The pet completely recovered from anaesthesia within 4.30 hours after induction. The owner was advised to keep the surgical site clean and apply povidone iodine ointment twice daily and Antibiotic with pain killer for 7 days. The pet recovered uneventfully and the skin suture removed on day 22 post surgery.

\section{Discussion and conclusion}

Open closed neuter is necessary for Boars as the testes descend at an early age. The inguinal ring remains relatively large throughout life so greatly increasing the risk of herniation. ${ }^{4}$ There are several probable reasons for the large number of losses guinea pigs are moreover sensitive to temperature, the abrupt change from $22^{\circ} \mathrm{C}$ to $16^{\circ} \mathrm{C}$ would tend to decrease their resistance to infection. Anaesthetic management in exotic species of animals is .always challenging due to the paucity of literature. Gowrdan \& Jimenez ${ }^{5}$ told that by intramuscular or intraperitoneal management of guinea pig at doess $0.1 \mathrm{~mL} / 100 \mathrm{~g}$ body weight of combination solution comprising of $5 \mathrm{~mL}$ of ketamine $(100 \mathrm{mg} / \mathrm{mL}), 2.5$ of $\mathrm{mL}$ xylazine $(20 \mathrm{mg} / \mathrm{mL}), 1 \mathrm{~mL}$ of acepromazine $(10 \mathrm{mg} / \mathrm{mL})$ and $1.5 \mathrm{~mL}$ of sterilized isotonic saline $(0.9 \%)$. Either Subcutaneous and intramuscular or intraperitoneal administration of anesthetic are ideal. In the present study, the anaesthetic protocol followed in the animal was found to be safe and the animal recovered rapidly (4.30 hours) from the anaesthesia. Animal should be kept on heating light or warm during surgery to ensure required body warmth. castration was approved out through a scrotal approach and testes could effortlessly be accessed and detached without any peri-operative complications. Angela ${ }^{6}$ indicated open closed technique of castration for small mammals including guinea pigs and it was reported that open closed technique of tip scrotal technique approach were found to be safe. Anderson \& Froimovitch ${ }^{4}$ blocked technique of castration in guinea pigs as there would be probability of herniation through the larger inguinal ring. It has been recommended to uphold the body temperature during surgery as they are sensitive to hypothermia. Different surgical techniques have been described for castration of guinea pigs: scrotal, prescrotal, and abdominal approach. ${ }^{7}$ The postsurgical scrotal incision should be closed and required post-operative therapy should be extremely maintained. In the present case, the anesthetic protocol followed in animal was found to be safe and the animal rapidly recovered. Castration was accepted through the scrotal approach and the testes could effortlessly be accessed and detached without any peri-operative complications and larger One Mild suppuration was noticed postoperatively 7 days and dressing was performed accordingly. The wound curative was noticed after 10 days of operation. ${ }^{8}$

\section{Post-operative cares}

As post-operative care, regular washing with $5 \%$ povicep ceftiaxone (Inj.ceftron ${ }^{\circledR}, 250 \mathrm{~mL}$ vial,) dosed at $30 \mathrm{mg} / \mathrm{kg}$ body weight (bwt), Meloxicam (Inj.Melvet, $10 \mathrm{~mL}$ vial,) dosed at $0.5 \mathrm{mg} / \mathrm{kg}$ bwt and Pheneramine meleate (Inj. Astavet, $10 \mathrm{~mL}$ vial, $22.75 \mathrm{mg} / \mathrm{mL}$ ) dosed at $1 \mathrm{mg} / \mathrm{kg}$ bwt were administered intramuscularly for seven days in 5day we found larger one abscess continue washing at 9 days we found good healed wound.

\section{Acknowledgments}

The authors are highly thankful to Prof. Bhajan Chandra Das, Department of Medicine and Surgery, Chittagong Veterinary and Animal Sciences University, Chittagong. Bangladesh for his inspiring advice and for the surgery.

\section{Conflicts of interest}

Authors declare that there is no conflict of interest.

\section{References}

1. Fraser T, Ascali RC. The castration of guinea pigs. J Inst Anim Techns. 1970;21:21-24.

2. Mcgreevy P. Handle with care. Halstead press: Sydney, Australia; 2002. $1-5 \mathrm{p}$

3. Richardson. In: Diseases of domestic guinea pigs. 2nd ed. Blackwell publications: USA; 2000. 14-15 p.

4. Anderson M, Froimovitch M. Simplified method of guinea pig castration. Can Vet J. 1974;15(4):126-127.

5. Gourdon J, Jimenez A. Guinea pig anesthesia. In: Comparative medicine and animal resources centre standard operative procedures; 2006 . 1-2 p.

6. Angela LM. There's more than one way to do it: Surgical castration techniques. Abvp (avian) and Exotic Clinic of Illinois, Indianapolis, Anavc Conference; 2008. 1824-1826 p.

7. Capello V. Common surgical procedures in pet rodents. Journal of Exotic Pet Medicine. 2011;20(4):294-307.

8. www.schools.nsw.edu.au/animalsinschools/index.htm. Animal welfare guidelines. 